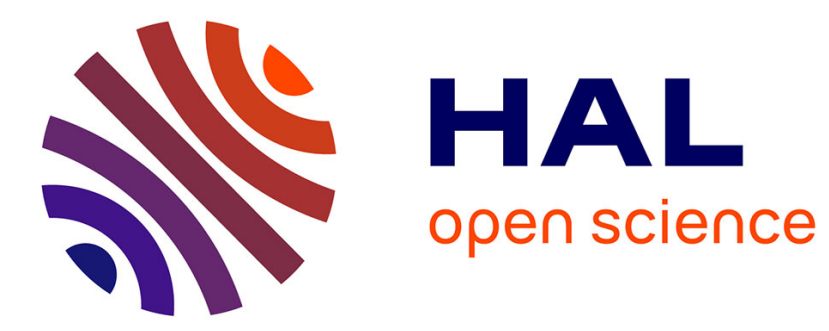

\title{
On the performance conditions of a N2 laser
}

P. Persephonis

\section{To cite this version:}

P. Persephonis. On the performance conditions of a N2 laser. Revue de Physique Appliquée, 1986, 21 (10), pp.609-617. 10.1051/rphysap:019860021010060900 . jpa-00245479

\section{HAL Id: jpa-00245479 https://hal.science/jpa-00245479}

Submitted on 1 Jan 1986

HAL is a multi-disciplinary open access archive for the deposit and dissemination of scientific research documents, whether they are published or not. The documents may come from teaching and research institutions in France or abroad, or from public or private research centers.
L'archive ouverte pluridisciplinaire HAL, est destinée au dépôt et à la diffusion de documents scientifiques de niveau recherche, publiés ou non, émanant des établissements d'enseignement et de recherche français ou étrangers, des laboratoires publics ou privés. 
Classification

Physics Abstracts

$42.55-52.70-35.80$

\title{
On the performance conditions of a $\mathbf{N}_{2}$ laser
}

\author{
P. Persephonis \\ Department of Physics, University of Patras, Patras 26110, Greece
}

(Reçu le 15 octobre 1985, revisé les 12 février et 19 juin 1986, accepté le 8 juillet 1986)

\begin{abstract}
Résumé. - On a étudié la puissance de pointe et le vrai voltage de départ des impulsions lumineuses du laser d'azote, en corrélation avec la pression et la fréquence de répétition des impulsions. Les résultats expérimentaux montrent que, sous des conditions appropriées de pression et de fréquence de répétition des impulsions, de grandes impulsions de puissance de pointe étaient émises. En comparant les résultats théoriques et expérimentaux, on donne des explications sur le comportement du plasma du laser et sur l'énergie émise pour diffẹrrentes valeurs de pression et la fréquence de répétition des impulsions. Par cette méthode, on peut estimer les coefficients de diffusion ambipolaires.
\end{abstract}

Abstract. - The peak power and the actual starting voltage of the $\mathrm{N}_{2}$ laser light pulses there have been studied, in relation to the pressure and the pulse repetition rate. The experimental results reveal that under proper conditions of pressure and pulse repetition rate, high peak power pulses are emitted. By comparing the theoretical and experimental results, there have been given explanations as far as the behaviour of the laser plasma and the emitted output energy for different values of pressure and pulse repetition rate is concerned. By this method the ambipolar diffusion coefficients can be estimated.

\section{Introduction.}

One of the most common lasers used at many applications is the nitrogen one. This is due mainly to the emission of a high power ultraviolet (UV) light pulse of short duration.

However some problems relative to its performance remain unsolved. These problems are mainly centred in the gas plasma behaviour. This plasma is created during a pulse discharge and it decays, through certain processes in the time interval between this pulse and the next one. After a discharge the remaining ions in the laser tube play the preionization role for the next one [1].

The external parameters through which the plasma can be controlled are :

(a) The pressure, (b) the pulse repetition rate, (c) the flow of the gas and (d) the applied voltage [2].

Unfortunately these external parameters are too few to control fully what exactly happens in the $\mathrm{N}_{2}$ plasma. Consequently, as it is expected, some statistical uncontrolled ununiformities in the plasma will cause different light output energy from pulse to pulse for the same values of the above mentioned external parameters.

The aim of this work is to find the optimal conditions under which the $\mathrm{N}_{2}$ laser could operate with the best stability and reliability.

\section{Experimental procedure-measurements.}

A Blumlein double-flat plat-transmission line TE $\mathrm{N}_{2}$ laser was used for the experiments of this work. Detailed description of this system is given in our previous works $[3,4]$. The equivalent electrical circuit is shown in figure 1 . The $\mathrm{N}_{2}$ laser system is triggered by a triggered spark-gap (EG \& G GP-70).

The laser tube was constructed of plexiglas and has a rectangular geometry with inner dimensions 4$4-40 \mathrm{~cm}$. (Its volume is $640 \mathrm{~cm}^{3}$ ). The gas flows transversely in the laser tube and is distributed uniformly in it. This is achieved by two parallel pipes placed along the laser tube so that the gas can get in and out through pinholes uniformly drilled. The rounded-off brass electrodes are $40 \mathrm{~cm}$ long, $0.5 \mathrm{~cm}$ wide and are $1.5 \mathrm{~cm}$ apart. Thus the discharge

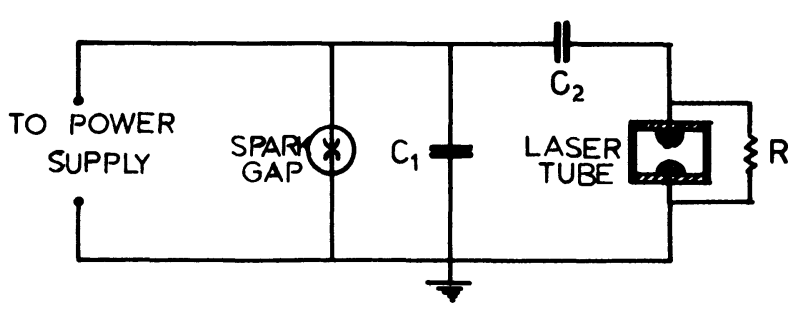

Fig. 1. - The equivalent electrical circuit of a Blumlein line $\mathrm{N}_{2}$ laser. 
volume is a part of the laser tube volume and it is situated far from the inner walls of the chamber. So the effects of parasitic preionization because of the charge development at the inner walls of the laser chamber through the Corona effect are avoided.

If the pressure in the laser tube is deliberately set too high then no break down in the tube will occur and the open-circuit output voltage of the pulse generator can be observed as it shown in figure 2 .

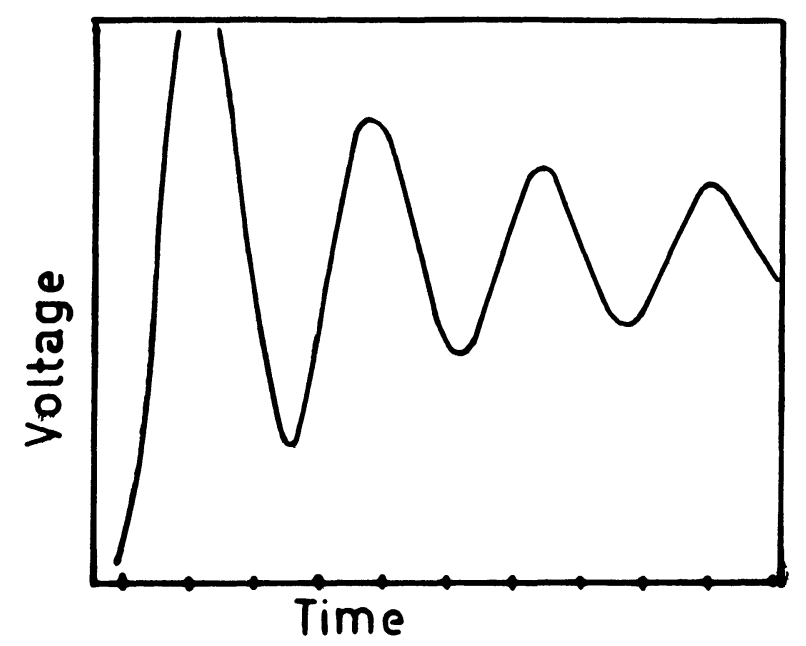

Fig. 2. - Open-circuit output voltage of the pulse generator. (Base time $50 \mathrm{~ns} / \mathrm{div}$ ).

By fitting the observed open-circuit output voltage with a damped sinusoidal oscillation of the form $V=V_{0}\left(1-\mathrm{e}^{-t / \tau} \cos \omega t\right)$ the damping time $\tau$ and the angular frequency $\omega$ can be determined. Their values are $\tau=247.9 \mathrm{~ns}$ and $\omega=46542.1 \times$ $10^{+3} \mathrm{~s}^{-1}$. By combining these values with the forms

$$
\omega=\left(L_{\mathrm{s}} C_{1}\right)^{-1 / 2} \text { and } R=\frac{2 L_{\mathrm{s}}}{\tau}
$$

the switch inductance $L_{\mathrm{s}}$ and the damping resistance $R$ can be estimated and they are :

$$
L_{\mathrm{s}}=57.764 \mathrm{nH} \text { and } R=0.466 \Omega \text {. }
$$

If the gas pressure in the laser tube is adjusted to be in the range of some tens of torr, the voltage across the laser tube increases following the waveform as shown in figure 2 . Then, as the discharge in the tube develops producing a highly conducting plasma the voltage stops to increase reaching a peak value $V_{\mathrm{s}}$ (actual starting voltage) and falls rapidly. This peak value of voltage depends on the pressure and on the initial ion density (preionization) $[5,6]$.

The optical output occurs at a time approximately halfway down to the falling laser tube voltage and increases by increasing the actual starting voltage for constant pressure. All these are shown in figure 3.

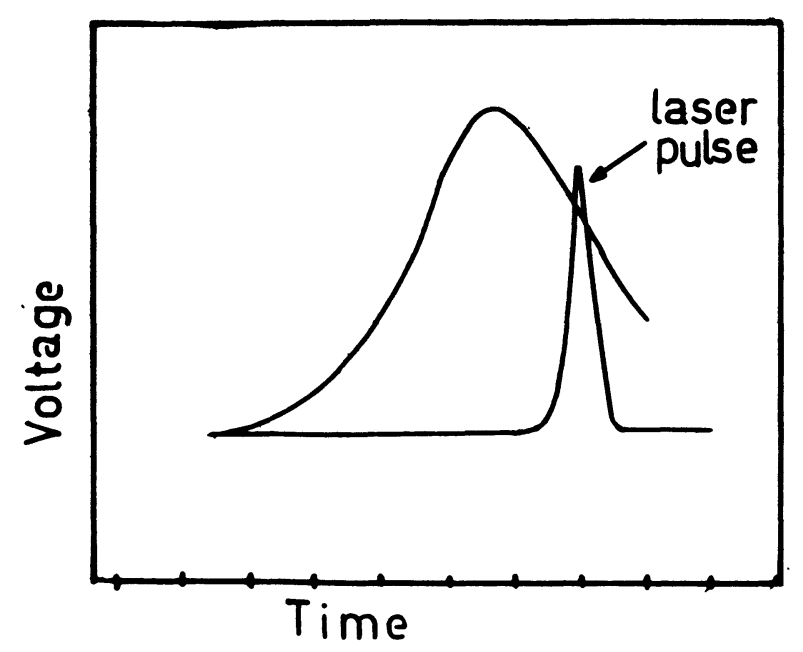

Fig. 3. - Optical output of the $\mathrm{N}_{2}$ laser relative to the voltage across the laser tube. (Base time $10 \mathrm{~ns} / \mathrm{div}$ ).

The measurements were carried out with a constant indication in our flowmeter $3.4 \mathrm{l} / \mathrm{min}$. this flowmeter is calibrated at $15^{\circ} \mathrm{C}$ and $760 \mathrm{~mm} \mathrm{Hg}$. For the above constant indication the flow rates at laser tube pressures are calculated according to the instructions of the manufacturer. Some of those are $0.78 \mathrm{1} / \mathrm{min}$ for 40 torr pressure ; $1.03 \mathrm{1} / \mathrm{min}$ for 70 torr $; 1.23 \mathrm{l} / \mathrm{min}$ for 100 and $1.4 \mathrm{l} / \mathrm{min}$ for 130 torr. The applied voltage was also constant $10 \mathrm{kV}$. The other two parameters (i.e. pressure and pulse repetition rate) were changed from 35 torr to 130 torr for the pressure and from $1.6 \mathrm{~Hz}$ to $25 \mathrm{~Hz}$, for the pulse repetition rate. These limits define the " domain" of the peak power and the actual starting voltage. This domain is shown in figure 4 . As we will see the most significant changes in the plasma take place in this domain.

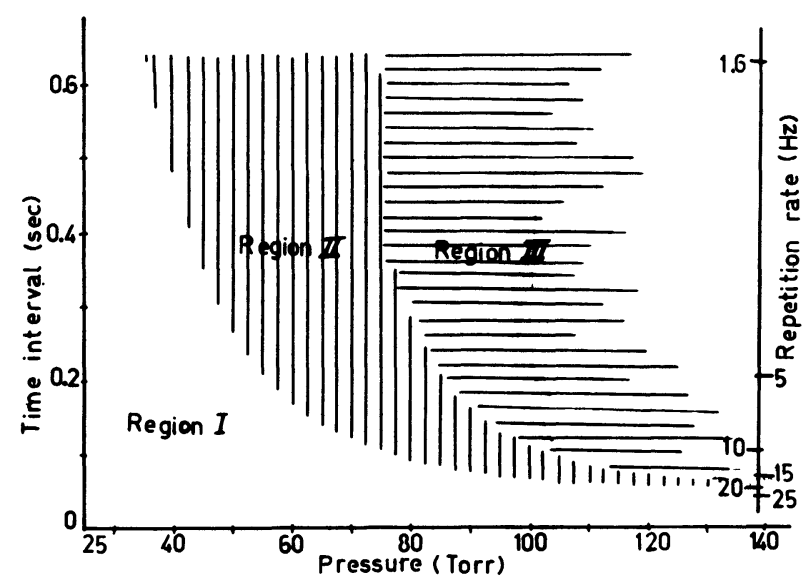

Fig. 4. - The " domain " of the peak power and the actual starting voltage. The domain is given as pressure versus time interval between two successive pulses $t_{\text {int }}$, $\left(t_{\text {int }}=\frac{1}{\nu}\right)$ where $\nu=$ pulse repetition rate. 
The laser beam was attenuated by a set of neutral density filters and detected by a fast photodiode (ITT F 4018). Also the actual starting voltage was detected by a high voltage probe (Tektronix P6015). The peak power and the actual starting voltage measurements have been taken simultaneously by a single beam multitrace display oscilloscope (Tektronix 7104).

All these measurements are shown in figures 5 and 6 for the actual starting voltage and the peak power respectively, while the calculated ratio $E / p$ is shown in figure 7.

Finally the efficiency of this system in terms of the optical energy per pulse divided by the stored electrical energy $\frac{1}{2} c V_{0}^{2}$ is about 0.00085 .

From the above measurements, one can draw two « general » conclusions.

(a) The actual starting voltage and the peak power increase by decreasing the pulse repetition rate in constant pressure.

(b) The actual starting voltage increases by increasing the pressure in constant pulse repetition rate.

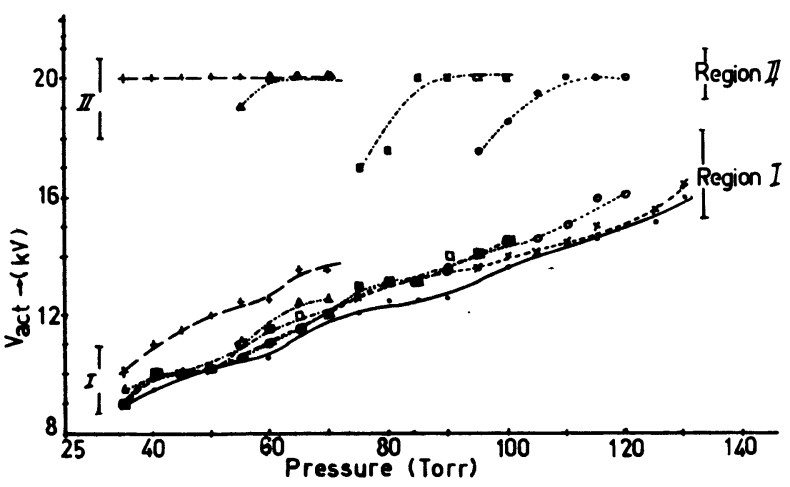

Fig. 5. - Actual starting voltage versus laser tube pressure, for different pulse repetition rates. $(+=1.6 \mathrm{~Hz}$, $\Delta=5 \mathrm{~Hz}, \quad \square=10 \mathrm{~Hz}, \quad O=15 \mathrm{~Hz}, \quad X=20 \mathrm{~Hz}, \quad 0=$ $25 \mathrm{~Hz}$.)

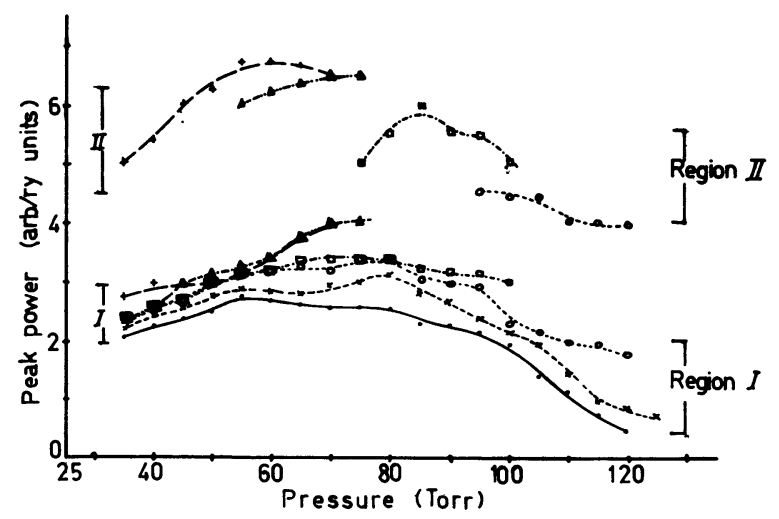

Fig. 6. - Laser peak power versus laser tube pressure, for different pulse repetition rate. $(+=1.6 \mathrm{~Hz}, \Delta=$ $5 \mathrm{~Hz}, \square=10 \mathrm{~Hz}, \mathrm{O}=15 \mathrm{~Hz}, \mathrm{X}=20 \mathrm{~Hz}, \Theta=25 \mathrm{~Hz}$.)

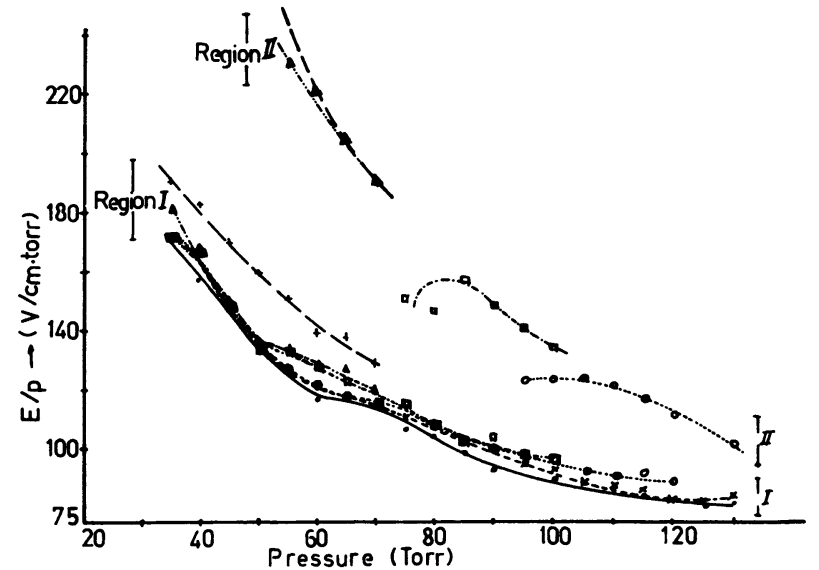

Fig. 7. $-E / p$ ratio versus laser tube pressure, for different pulse repetition rate. $(+=1.6 \mathrm{~Hz}, \Delta=5 \mathrm{~Hz}, \square=$ $10 \mathrm{~Hz}, \mathrm{O}=15 \mathrm{~Hz}, \mathrm{X}=20 \mathrm{~Hz}, \bigcirc=25 \mathrm{~Hz}$.)

On the other hand the peak power in constant pulse repetition rate increases by increasing the pressure in low pressure regions, while in high pressure regions this decreases. So each curve of peak power versus pressure in figure 6 forms a large maximum centred in a certain pressure. These pressure values increase as the pulse repetition rate decreases.

One of the results of the experimental work was the finding of a region in the above defined domain, where the actual starting voltage and consequently the peak power of the light pulses get rather very high values with a jitter about $15 \%$ for both quantities. These values of the actual starting voltage, as shown in figure 5 , approach the $20 \mathrm{kV}$ while the values of the peak power, as shown in figure 6 , are nearly double as to those in the other region of this domain. The ratio $E / p$ takes very high values in this region and the electron temperature too (see Fig. 7).

There is also one more region in this domain where no break down in the tube occurs and the $\mathrm{N}_{2}$ laser stops to emit light pulses.

Conclusively : three regions are distinguished in the domain. The region (I), where the actual starting voltage and peak power values are sufficiently stable (nearly without jitter). The region (II), where the actual starting voltage and peak power values are unstable (with jitter). These values are very high relative to the ones of the region I (see Figs. 5 and 6 ). The region (III) where the $\mathrm{N}_{2}$ laser stops to emit light pulses because no gas break down occurs. In this region there have been taken the above mentioned measurements of the open-circuit output voltage. The three regions of the domain are shown in figure 4 .

It is worth emphasizing that the boundaries of these three regions, in figure 4, are not distinct. In these boundary areas some of the pulses belong to those pulses which correspond to one region while the remainders belong to the kind of pulses which correspond to the neighbouring region. This possibly 
causes the illusion that a very big jitter exists in these boundary areas. As we go from one region to another the frequency of the appearance of the kind of pulses, which correspond to the region we leave decreases until it is annihilated. The above remarks are valid for both the actual starting voltage and the light intensity as well.

These regions are also distinguished in the laser tube, observing the plasma by nude eye. Thus when the laser operates under conditions of region I the plasma in the laser tube has the visual appearance of a thin uniform band connecting the electrodes, whereas under conditions of region II the brightness of the plasma band becomes ununiform with appearance of filaments.

Finally a jitter in the light pulses was observed, at high pressures and high pulse repetition rates. The more the repetition rate is increased the more it increases. No simultaneous jitter in the actual starting voltage was observed. This will be explained later.

\section{Theoretical considerations on the electrical pro- perties of a $\mathbf{N}_{2}$ laser.}

We consider that the theoretical treatment of a $\mathrm{N}_{2}$ laser by Fitzsimmons et al. [7] is in good agreement with the reality and that the referred relationships in their work are valid. These are as follows :

The drift velocity $U_{\mathrm{d}}$ and the Townsend coefficient divided by the pressure $\alpha / p$ are function of $E / p$ so

$$
\begin{aligned}
U_{\mathrm{d}} & =2.9 \times 10^{5}(E / p) \mathrm{cm} / \mathrm{s} \\
\alpha / p & =1.4 \times 10^{-8}(E / p)^{3.7}(\text { torr } . \mathrm{cm})^{-1} .
\end{aligned}
$$

The current flow in the laser tube is governed by the equations

$$
\begin{gathered}
L \frac{\mathrm{d} I}{\mathrm{~d} T}+I\left(R_{\mathrm{g}}+Z_{0}\right)=V_{\mathrm{eff}} \\
V_{\text {eff }}=V_{0}\left(1-\mathrm{e}^{-t / \tau} \cos \omega t\right)-\frac{2}{C_{1}} \int_{0}^{t} I \mathrm{~d} t
\end{gathered}
$$

Where $V_{0}=$ initial charge voltage

$L=$ inductance of the load (laser tube)

$R_{\mathrm{g}}=$ time dependent resistance of the laser discharge

$Z_{0}=$ characteristic impedance of the transmission line

$V_{\text {eff }}=$ the measured open-circuit voltage of the voltage-pulse generator minus a term that represents the discharge of the Blumlein capacitance.

The multiplication of the electron density in the laser tube is given by the Townsend equation $\mathrm{d} n / \mathrm{d} t=\alpha U_{\mathrm{d}} n$. Integration of this equation gives $n$ as a function of time.
The conductivity of the gas as a function of time is given by

$$
\sigma=\frac{e n U_{\mathrm{d}}}{E}
$$

Assuming a uniform electric field between the laser tube electrodes the resistance of the discharge is

$$
R_{\mathrm{g}}=\frac{d}{s \cdot w \cdot \sigma}
$$

where $d$ is the electrode separation, $s$ is the thickness of the arc and $w$ is the active length of the laser tube. The current is given by the form $I=n e U_{\mathrm{d}}(s \cdot w)$. Finally the electric field between the electrodes as a function of time is given by

$$
E=\frac{1}{d}\left(I R_{\mathrm{g}}+L \frac{\mathrm{d} I}{\mathrm{~d} t}\right) .
$$

Numerical simultaneous solution of these equations was carried out using a digital computer. However the solution of these equations requires to accept an initial ion density at the start of the calculation. This is because different initial ion densities give different solutions for the same pressure.

By combining the above equations one can see that the uniqueness of the solution concerning with the obtained initial ion densities requires the uniqueness of the electric field $E$. This is a macroscopic quantity the uniqueness of which is indicated by the experimental measurements.

In table I the calculated values of the actual starting voltage for different pressures and different initial ion density are given.

When a discharge in a $\mathrm{N}_{2}$ laser tube takes place with an initial ion density $n_{0}$, then the ion density, by the avalanche multiplication of ions, gets to the maximum value $n_{\max }$, nearly at the end of the discharge. Then the ion population decays through certain processes in the time interval between this pulse and the next one.

Here it must be emphasized that the flow rate of the gas is too small to contribute to the ions removal from the laser tube. In a time of $0.1 \mathrm{~s}$ only the $8.85 / 1000$, of the laser tube volume is renewed by the flow of the gas in atmospheric pressure ; while at laser tube pressures this fraction becomes smaller, for example in 70 torr (around this pressure the large maxima of the curves peak power versus pressure in figure 6 are formed) is 2.68/1 000. The discharge volume is mainly situated among the electrodes. Its value can be found as a product of its cross section multiplied by the electrodes length. The cross section of this volume is the same with the one of the laser light beam immediately after its exit from the laser tube. Thus the discharge volume can be estimated about $45 \mathrm{~cm}^{3}$. In a time of $0.1 \mathrm{~s}$ for example in 70 torr pressure only the $38.1 / 1000$ of the discharge volume is renewed by the flow of the gas. In the worst case the biggest time interval between two 
Table I. - Calculated values of the actual starting voltage for different pressures and different initial ion densities. $(+=1.6 \mathrm{~Hz}, \Delta=5 \mathrm{~Hz}, \square=10 \mathrm{~Hz}, \mathrm{O}=15 \mathrm{~Hz}, X=20 \mathrm{~Hz}, \quad=25 \mathrm{~Hz}$.)

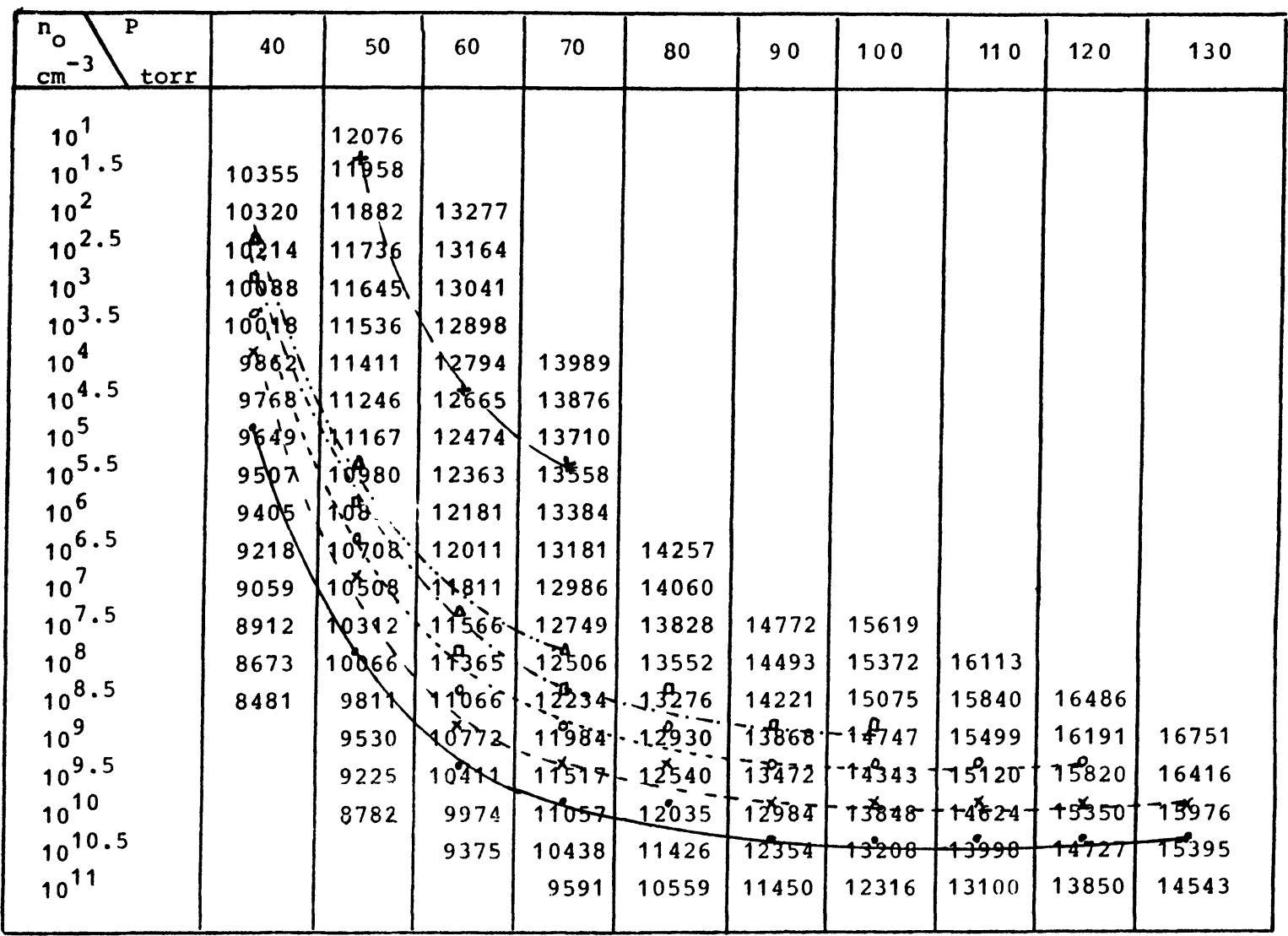

successive pulses in 70 torr pressure and for stable pulses (region I) is $0.12 \mathrm{~s}$ as can be seen in figure 4 . In that time the renewal fraction of the discharge volume becomes only $45.7 / 1000$. For shorter time intervals (higher repetition rates) this fraction becomes significantly smaller. Moreover one must bear in mind that immediately after the discharge the high temperature ions and electrons in the discharge volume are diffused at high speeds (greater than the flow speed of the gas) to all directions. However the mean « diffusion time » is about a few (1-10) milliseconds [15-17]. That is, the most of the ions are diffused in this order of time to the whole of the laser tube volume. Consequently the flow of the gas simply brings the diffused ions back to the discharge volume. Thus we can say that the gas flow does not contribute to the removal of the ions from the discharge volume.

Consequently the ion losses must be attributed mainly to certain processes (diffusion, recombination, etc.). These processes will be discussed later. The flow of the gas serves mainly to remove the heat from the laser tube. The density of the remaining ions after the decay constitutes the initial ion density for the next pulse. In table II the calculated values of the maximum ion density for different pressure and different initial ion density are given.

Moreover, when the $\mathrm{N}_{2}$ laser operates in the region I of the domain (Fig. 4) the pulses are stable (nearly without jitter from pulse to pulse) in constant pressure and pulse repetition rate. The conditions under which we have stable pulses require the initial ion density to be the same for every pulse. This means that the number of the created ions during the discharge must be equal to the number of the decaying ones after the discharge so that the initial ion density remains the same. This requirement is satisfied under proper pairs of values of the pressure and the pulse repetition rate. These pairs of values compose the region I of the domain. The ranges of the actual starting voltage and of the peak power for this region of the domain are shown in figures 5 and 6 respectively.

If these experimental values of the actual starting voltage (for stable pulses) are recognized and are checked among the calculated actual starting voltage ones in table I for every pressure then one can read the initial ion density on the left of the table for every checked value of the actual starting voltage. The drawn curves in table I join the checked values of the actual starting voltage for the same pulse repetition rate. Also in table II one can check the calculated maximum ion density values corresponding to the actual starting voltage values checked in table I.

The drawn curves in table II join the maximum ion density checked values for the same pulse repetition rate too. 
Table II. - Calculated values of maximum ion density $n_{\max }\left(n_{\max }=\right.$ indication $\left.\times 10^{14}\right)$, for different pressures and different initial ion densities $n_{0} .(+=1.6 \mathrm{~Hz}, \Delta=5 \mathrm{~Hz}, \square=10 \mathrm{~Hz}, O=15 \mathrm{~Hz}, \mathrm{X}=20 \mathrm{~Hz}, \bullet=25 \mathrm{~Hz}$.)

\begin{tabular}{|c|c|c|c|c|c|c|c|c|c|c|}
\hline $\begin{array}{l}\mathrm{n}_{0} \\
\mathrm{~cm}^{-3} \text { torr }^{\mathrm{P}}\end{array}$ & 40 & 50 & 60 & 70 & 80 & 90 & 100 & 110 & 120 & 130 \\
\hline $\begin{array}{l}10^{1} \\
10^{1.5} \\
10^{2} \\
10^{2.5} \\
10^{3} \\
10^{3.5} \\
10^{4} \\
10^{4.5} \\
10^{5} \\
10^{5.5} \\
10^{6} \\
10^{6.5} \\
10^{7} \\
10^{7.5} \\
10^{8} \\
10^{8.5} \\
10^{9} \\
10^{9.5} \\
10^{10} \\
10^{10.5} \\
10^{11}\end{array}$ & $\begin{array}{l}1.42 \\
1.41 \\
1442 \\
19.47 \\
19.49 \\
1.36 \\
1.38 ! \\
1.36 \\
1.30 \\
1.37 \\
1.32 \\
1.29 \\
1.23 \\
1.25 \\
1.18\end{array}$ & $\begin{array}{l}1.72 \\
1+74 \\
1.70 \\
1.73 \\
1.77 \\
1.68 \\
1.67 \\
1.66 \\
1.61 \\
1463 \\
19451 \\
1959\end{array}$ & $\begin{array}{l}2.02 \\
2.01 \\
1.93 \\
1.95 \\
1.97 \\
1.93 \\
1.90 \\
1.89 \\
1.90 \\
1.85 \\
.83\end{array}$ & $\begin{array}{l}2.24 \\
2.15 \\
2.18 \\
2.73 \\
2.10 \\
2.11 \\
2.05 \\
2.04 \\
7.97 \\
1.99 . \\
1.89 \\
1.89 \\
1.86 \\
1.72 \\
1.58\end{array}$ & $\begin{array}{l}2.27 \\
2.30 \\
2.26 \\
2.16 \\
2.16 \\
2.15 \\
2 \times 04 \\
2904 \\
1.87 \\
1.73\end{array}$ & $\begin{array}{c}2.47 \\
2.49 \\
2.35 \\
2.34 \\
-2.03 \\
-2.25 \\
-2.10 \\
1.98\end{array}$ & $\begin{array}{r}2.72 \\
2.62 \\
2.64 \\
-2.50 \\
-2.452 \\
-2.0 .32- \\
20.22 \\
2.15\end{array}$ & $\begin{array}{r}2.89 \\
2.81 \\
2.74 \\
-2.73^{-1} \\
-2 \times 54 \\
2.52 \\
2.37\end{array}$ & $\begin{array}{c}2.92 \\
2.97 \\
20.71 \\
2.7 \% 66 \\
2.64 \\
2.41\end{array}$ & $\begin{array}{r}3.10 \\
2.85 \\
-2.882 \\
-2.79 \\
2.59\end{array}$ \\
\hline
\end{tabular}

Finally in the table III the initial ion densities $n_{0}$ and the maximum ion densities $n_{\max }$ of all the actual starting voltage values checked in table I (i.e. values of stable pulses) are given. These ion density values have been transferred in this table from the table II.

Table III. - The initial ion densities $n_{0}$ and the maximum ion densities $n_{\max }$ of all the checked actual starting voltage values in table I.

\begin{tabular}{|c|c|c|c|c|c|c|c|c|c|c|c|}
\hline $\mathrm{n}_{\mathrm{r}}$ & 40 torr & 50 torr & 60 torr & 70 torr & 80 torr & 90 torr & 100 torr & 110 torr & 120 torr & 130 torr & $\left.\begin{array}{l}\mathrm{n}_{0} \\
\mathrm{n}_{\max } \\
\mathrm{cm}^{-3}\end{array}=\frac{1}{v}\right)$ \\
\hline 1.6 & & $\begin{aligned} \mathrm{n}_{0}= & 10^{1.5} \\
\mathrm{n}_{\max }= & 10^{14} \times \\
& \times 1.74\end{aligned}$ & $\begin{aligned} \mathrm{n}_{0}=10^{4.5} \\
\mathrm{n}_{\max }=10^{14} \times \\
\times 1.93\end{aligned}$ & $\begin{aligned} \mathrm{n}_{0}=10^{5.5} \\
\mathrm{n}_{\max }=10^{14} \times \\
\times 2.13\end{aligned}$ & & & & & & & 0.625 \\
\hline 5 & $\begin{aligned} \mathrm{n}_{0}=10^{2.5} \\
\mathrm{n}_{\max }=10^{14} \times \\
\times 1.42\end{aligned}$ & $\begin{aligned} \mathrm{n}_{0}= & 10^{5.5} \\
\mathrm{n}_{\max }= & 10^{14} \times \\
& \times 1.64\end{aligned}$ & $\begin{aligned} \mathrm{n}_{0}= & 10^{7.5} \\
\mathrm{n}_{\max }= & 10^{14} \times \\
& \times 1.76\end{aligned}$ & $\begin{aligned} \mathrm{n}_{0}= & 10^{8} \\
\mathrm{n}_{\max }= & 10^{14} \times \\
& \times 1.97\end{aligned}$ & & & & & & & 0.2 \\
\hline 10 & $\begin{aligned} \mathrm{n}_{0}= & 10^{3} \\
\mathrm{n}_{\max }= & 10^{14} \times \\
& \times 1.37\end{aligned}$ & $\begin{aligned} \mathrm{n}_{0}= & 10^{6} \\
\mathrm{n}_{\max }= & 10^{14} \times \\
& \times 1.61\end{aligned}$ & $\begin{aligned} \mathrm{n}_{0}= & 10^{8} \\
\mathrm{n}_{\max }= & 10^{14} \times \\
& \times 1.72\end{aligned}$ & $\begin{aligned} \mathrm{n}_{0}= & 10^{8.5} \\
\mathrm{n}_{\max }= & 10^{14} \times \\
& \times 1.99\end{aligned}$ & $\begin{aligned} \mathrm{n}_{0}= & 10^{8.5} \\
\mathrm{n}_{\max }= & 10^{14} \times \\
& \times 2.16\end{aligned}$ & $\begin{array}{l}\mathrm{n}_{0}=10^{9} \\
\mathrm{n}_{\max }=10^{14} \times \\
\times 2.34\end{array}$ & $\begin{aligned} & \mathrm{n}_{0}= 10^{9} \\
& \mathrm{n}_{\max }= 10^{14} \times \\
& \times 2.50\end{aligned}$ & & & & 0.1 \\
\hline 15 & $\begin{aligned} & \mathrm{n}_{0}= 10^{3.5} \\
& \mathrm{n}_{\max }= 10^{14} \times \\
& \times 1.39\end{aligned}$ & $\begin{aligned} \mathrm{n}_{0}= & 10^{6.5} \\
\mathrm{n}_{\max }= & 10^{14} \times \\
& \times 1.59\end{aligned}$ & $\begin{aligned} & \mathrm{n}_{0}=10^{8.5} \\
& \mathrm{n}_{\max }= 10^{14} \times \\
& \times 1.75\end{aligned}$ & $\begin{aligned} \mathrm{n}_{0}=10^{9} \\
\mathrm{n}_{\max }=10^{14} \times \\
\times 1.87\end{aligned}$ & $\begin{array}{l}\mathrm{n}_{0}=10^{9} \\
\mathrm{n}_{\max }=10^{14} \times \\
\times 2.15\end{array}$ & $\begin{aligned} \mathrm{n}_{0}= & 10^{9.5} \\
\mathrm{n}_{\max }= & 10^{14} \times \\
& \times 2.23\end{aligned}$ & $\begin{aligned} \mathrm{n}_{0}= & 10^{9.5} \\
\mathrm{n}_{\max }= & 10^{14} \times \\
& \times 2.42\end{aligned}$ & $\begin{aligned} \mathrm{n}_{0}= & 10^{9.5} \\
\mathrm{n}_{\max }= & 10^{14} \times \\
& \times 2.73\end{aligned}$ & $\begin{aligned} \mathrm{n}_{0}= & 10^{9.5} \\
\mathrm{n}_{\max }= & 10^{14} \\
& \times 2.71\end{aligned}$ & & 0.0666 \\
\hline 20 & $\begin{aligned} & \mathrm{n}_{0}=10^{4} \\
& \mathrm{n}_{\max }= 10^{14} \times \\
& \times 1.36\end{aligned}$ & $\begin{aligned} \mathrm{n}_{0}= & 10^{7} \\
\mathrm{n}_{\max }= & 10^{14} \times \\
& \times 1.53\end{aligned}$ & $\begin{aligned} \mathrm{n}_{0}= & 10^{9} \\
\mathrm{n}_{\max }= & 10^{14} \times \\
& \times 1.67\end{aligned}$ & $\begin{aligned} \mathrm{n}_{0}= & 10^{9.5} \\
\mathrm{n}_{\max }= & 10^{14} \times \\
& \times 1.89\end{aligned}$ & $\begin{aligned} \mathrm{n}_{0}= & 10^{9.5} \\
\mathrm{n}_{\max }= & 10^{14} \times \\
& \times 2.04\end{aligned}$ & $\begin{aligned} \mathrm{n}_{0}= & 10^{10} \\
\mathrm{n}_{\max }= & 10^{14} \times \\
& \times 2.25\end{aligned}$ & $\begin{aligned} \mathrm{n}_{0}= & 10^{10} \\
\mathrm{n}_{\max }= & 10^{14} \times \\
& \times 2.32\end{aligned}$ & $\begin{aligned} \mathrm{n}_{0}= & 10^{10} \\
\mathrm{n}_{\max }= & 10^{14} \times \\
& \times 2.54\end{aligned}$ & $\begin{aligned} \mathrm{n}_{0}= & 10^{10} \\
\mathrm{n}_{\max }= & 10^{14} \times \\
& \times 2.66\end{aligned}$ & $\begin{aligned} \mathrm{n}_{0}= & 10^{10} \\
\mathrm{n}_{\max }= & 10^{14} \times \\
& \times 2.82\end{aligned}$ & 0.05 \\
\hline 25 & $\begin{aligned} \mathrm{n}_{0}= & 10^{5} \\
\mathrm{n}_{\max }= & 10^{14} \times \\
& \times 1.36\end{aligned}$ & $\begin{aligned} \mathrm{n}_{0}= & 10^{8} \\
\mathrm{n}_{\max }= & 10^{14} \times \\
& \times 1.49\end{aligned}$ & $\begin{aligned} \mathrm{n}_{0}= & 10^{9.5} \\
\mathrm{n}_{\max }= & 10^{14} \times \\
& \times 1.60\end{aligned}$ & $\begin{aligned} \mathrm{n}_{0}= & 10^{10} \\
\mathrm{n}_{\max }= & 10^{14} \times \\
& \times 1.76\end{aligned}$ & $\begin{array}{l}\mathrm{n}_{0}=10^{10} \\
\mathrm{n}_{\max }=10^{14} \times \\
\times 2.04\end{array}$ & $\begin{aligned} \mathrm{n}_{0}= & 10^{10.5} \\
\mathrm{n}_{\max }= & 10^{14} \times \\
& \times 2.10\end{aligned}$ & $\begin{aligned} \mathrm{n}_{0}= & 10^{10.5} \\
\mathrm{n}_{\max }= & 10^{14} \times \\
& \times 2.22\end{aligned}$ & $\begin{aligned} \mathrm{n}_{0}= & 10^{10.5} \\
\mathrm{n}_{\max }= & 10^{14} \times \\
& \times 2.52\end{aligned}$ & $\begin{aligned} \mathrm{n}_{0}= & 10^{10.5} \\
\mathrm{n}_{\max }= & 10^{14} \times \\
& \times 2.61\end{aligned}$ & $\begin{array}{c}\mathrm{n}_{0}=10^{10.5} \\
\mathrm{n}_{\max }=10^{14} \times \\
2.79\end{array}$ & 0.04 \\
\hline
\end{tabular}


The values of the initial and maximum ion density in table III which correspond to the boundary zone between the regions I and II where both kinds of pulses appear have been taken from those pulses which correspond to region I. It is also noted that for each measurement of the actual starting voltage of the pulses which correspond to the region I the control of the plasma uniformity observed by nude eye was indispensable.

\section{Ion decay mechanisms.}

As it is known, the actual starting voltage increases by decreasing the initial ion density [5-7].

This is achieved mainly by decreasing the pulse repetition rate, that is to say, by increasing the time interval between two pulses, so that the decaying ions increase and the remaining ones decrease.

As mentioned above, in the region $I$ of the domain (Fig. 4) the stable pulses require stable values of the initial and maximum ion density. This implies that the mechanism which governs the ion decay in this region does not allow fluctuations in the final ion density and secures the stability of the light pulses. This mechanism and generally the microscopic phenomena which take place in this plasma will be discussed later.

On the other hand the main feature of the region II is the emission of light pulses with high values of peak power relative to the ones in region I, which correspond to high values of the actual starting voltage (nearly at the top of the waveform in Fig. 2). These high indensity light pulses are attributed to another decay ion process predominant in this region. By this process the ion density decreases very quickly to very small values (approaching to zero). The few remaining ions are the cause of these high indensity light pulses in this region of the domain. Yet the existence of jitter in these pulses implies that the predominant at this region ion decay process permits statistical fluctuations in the ion density values from pulse to pulse.

Finally in the region III of the domain the break down in the tube does not occur and the $\mathrm{N}_{2}$ laser stops to emit light pulses. This is attributed to the fact that the same ion decay process continues to predominate in the region III as well. This decay process continues to evacuate the tube from the ions so the remaining ones are too few to cause break down in the gas.

Consequently one can say the following about the ion decay in the laser tube.

Immediately after the discharge the plasma begins to decay by the predominant, ion decay process, in region I. Then after a certain time different to any value of pressure, the ion decay process referred in region II begins to predominate now, while the previous ion decay process retreats. In the boundary between the two regions both ion decay processes are important (Fig. 4).

The above described ion decay which began after a pulse discharge is interrupted by the next pulse. The time interval $\left(t_{\text {int }}\right)$ between two pulses and consequently the repetition rate $\left(t_{\text {int }}=\frac{1}{\nu}\right)$ determines the "situation" of the remaining ions from a discharge at the beginning of the next one. This time interval defines (for a given pressure) the region of the domain where the $\mathrm{N}_{2}$ laser operates.

So for $t_{\text {int }}<t_{\mathrm{b}}(\mathrm{I}, \mathrm{II})$ the $\mathrm{N}_{2}$ laser operates in the region $\mathrm{I}$,

for $t_{\mathrm{b}}(\mathrm{I}, \mathrm{II})<t_{\text {int }}<t_{\mathrm{b}}$ (II, III) the $\mathrm{N}_{2}$ laser operates in the region II,

and for $t_{\mathrm{b}}$ (II, III) $<t_{\text {int }}$ the $\mathrm{N}_{2}$ laser operates in the region III.

Where the $t_{\mathrm{b}}$ (I, II) and $t_{\mathrm{b}}$ (II, III) are the boundary times (for a given pressure) of the regions I and II and of the regions II and III respectively.

It is known that there are two fundamental decay processes occurring in a plasma after a gas discharge. These are the diffusion and the recombination.

In the general case where both diffusion and recombination are playing an important role in the plasma decay the continuity equation becomes

$$
\frac{\partial n}{\partial t}=-\alpha n^{2}+D_{\alpha} \nabla^{2} n
$$

where $\quad \alpha=$ Recombination coefficient

$$
D_{\alpha}=\text { Ambipolar diffusion coefficient. }
$$

This is a nonlinear equation and so no simple solution is available. In some cases however approximate solutions can be found. For instance if the recombination is small with respect to diffusion, we can assume a fundamental diffusion mode with a decay time $\tau$ and rewrite $[8,9]$

$$
\frac{\partial n}{\partial t}=-\alpha n^{2}-\frac{n}{\tau}
$$

An approximate solution to this equation is

$$
\frac{n}{1+\alpha \tau n}=\frac{n_{0}}{1+\alpha \tau n_{0}} \cdot \mathrm{e}^{-t / \tau} \text {. }
$$

In the more general case, however, it is necessary to use a more sophisticated analysis [10].

When the only ion decay process is the diffusion then the equation (1) degenerates to

$$
\frac{\partial n}{\partial t}=D_{\alpha} \nabla^{2} n \text { or } \frac{\partial n}{\partial t}=-\frac{n}{\tau}
$$

(from Eq. (2)) and the solution is given by the equation [11]

$$
n=n_{0} \mathrm{e}^{-t / \tau} .
$$

On the other hand when the only ion decay process is the recombination the equation (1) degenerates to

$$
\frac{\partial n}{\partial t}=-\alpha n^{2}
$$


and the solution is given by the equation [12-14]

$$
\frac{1}{n}=\frac{1}{n_{0}}+\alpha t
$$

Also as it is known the diffusion coefficient decreases by increasing the gas pressure while the recombination coefficient increases by increasing the gas pressure [15]. Consequently recombination is a particularly important deionization process at high pressures for which diffusion is slow. At low pressures diffusion usually becomes more important.

The fundamental nature of the recombination process depends on the concentration of both the ions and the neutral molecules. The ions are responsible for the Coulomb forces active and the neutralparticle density governs the frequency of collisions and the mean free path in the gas.

If we consider two charges of opposite polarities and assume that one of them is stationary with respect to the other, then the second particle will be accelerated towards the first. This condition can be expressed by equating its potential energy with its kinetic energy [16].

$$
\frac{3}{2} K T=\frac{e^{2}}{4 \pi \varepsilon_{0} d} .
$$

When the kinetic energy exceeds the potential energy the ion will not be affected by the field of the stationary ion. By the equation (8) it is possible to find the "volume of active attraction" which is given by a "sphere of influence " the radius $d$ of which is the distance at which the electrostatic force ceases to be active against the forces resulting from thermal agitation. So $d$ is

$$
d=\frac{e^{2}}{6 \pi \varepsilon_{0} K T} .
$$

If the average distance between the ions $(\alpha)$ is lesser than $d$, the forces due to the ion electric fields will prevail. Then the ions collide and recombine. $\alpha$ can be obtained simply from

$$
\alpha=\left(\frac{1}{n}\right)^{1 / 3}
$$

where $n$ is the initial ionic concentration of the positive ions at $t=0$.

If, on the other hand, $\alpha$ is much larger than $d$, the ions must undergo diffusion before they are « trapped " in the active sphere of one of the opposite ions and recombine [16].

In our case the calculated $\alpha$ by the data of the table III is at least three orders of magnitude larger than the calculated $d$ in equation (9). So the initial ion decay process after the discharge is the diffusion. The recombination follows. Consequently the predominant ion decay process in the region I must be the diffusion, while in the region II must be the recombination (Fig. 4).
The microscopic phenomena which take place in the plasma are the following.

The high voltage discharge permits the passage of a high current through the gas and the injection of energy sufficiently large into the discharge volume during a very small time. This causes the creation of ions and excited molecules. The population inversion which takes place between the $\mathrm{C}^{3} \Pi_{\mathrm{u}}$ and $\mathrm{B}^{3} \Pi_{\mathrm{g}}$ states produces the lasing action. Immediately after the discharge the existing high temperature ions, electrons and excited molecules in the discharge volume diffuse at high speeds (greater than the flow speed of the gas) and consequently the gas flow does not contribute to the removal of the ions from the discharge volume. Then the diffused ions and electrons interact. The collisions among ions give ionic reactions [2] while the collisions between ions and electrons give recombinations.

Using the equation (3) and the data of the table III, the values of the " mean diffusion decay time " in the region I can be calculated. These are shown in figure 8 . The recombination coefficient in the region $I$ is also calculated and it is of the order of $10^{-12} \mathrm{~cm}^{3} \mathrm{~s}^{-1}$, while in the region II the coefficient must be of the order of $10^{-6}-10^{-7} \mathrm{~cm}^{3} \mathrm{~s}^{-1}$ [12-14]. This very small value of the recombination coefficient in the region I indicates that this ion decay process in the region I is nearly nonexistent. This also becomes more evident when one considers only the diffusion ion decay process and using the equation (5) recalculates the values of the diffusion decay time. These values are nearly the same as the calculated ones previously by the equation (3) and are shown in figure 8.

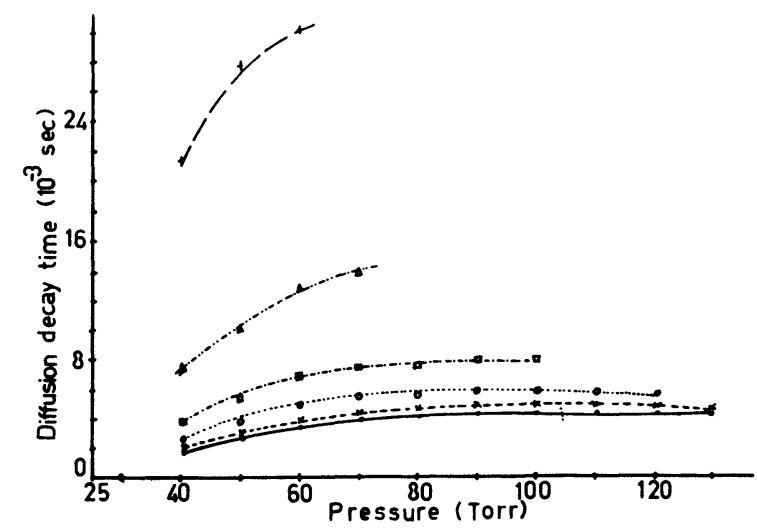

Fig. 8. - Diffusion decay time versus laser tube pressure, for different pulse repetition rate. $(+=1.6 \mathrm{~Hz}, \Delta=$ $5 \mathrm{~Hz}, \square=10 \mathrm{~Hz}, \mathrm{O}=15 \mathrm{~Hz}, \mathrm{X}=20 \mathrm{~Hz}, \bullet=25 \mathrm{~Hz}$.)

The mean ambipolar diffusion coefficient can be found by the relation [8]

$$
\Lambda=\sqrt{D_{\alpha} \tau}
$$

where $\Lambda$ is the " characteristic diffusion length ». 
This is given by the relation [17]

$$
\frac{1}{\Lambda^{2}}=\left(\frac{\pi}{L_{1}}\right)^{2}+\left(\frac{\pi}{L_{2}}\right)^{2}+\left(\frac{\pi}{L_{3}}\right)^{2}
$$

for rectangular geometry of the diffusion space. In our case $\Lambda=0.337 \mathrm{~cm}$. By using the relation (11) the calculated valvues of the ambipolar diffusion coefficient are shown in figure 9. In this figure we observe that the ambipolar diffusion coefficient (for a given pressure) decreases by decreasing the pulse repetition rate, that is to say, by increasing the time interval between two pulses. This indicates that after the discharge the predominant diffusion process retreats by the time. Yet the ambipolar diffusion coefficient values, shown in figure 9 , are of the same order of magnitude with the existent values in the literature $[16,18]$.

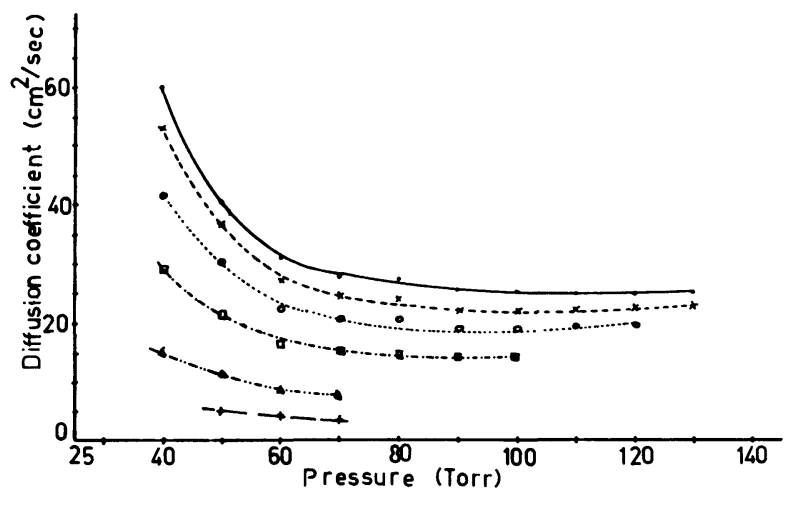

Fig. 9. - Ambipolar diffusion coefficient versus laser tube pressure, for different pulse repetition rate. $(+=1.6 \mathrm{~Hz}, \Delta=5 \mathrm{~Hz}, \quad \square=10 \mathrm{~Hz}, O=15 \mathrm{~Hz}, X=$ $20 \mathrm{~Hz}, \bigcirc=25 \mathrm{~Hz}$.)
This agreement indicates that the work of Fitzsimmons et al. [7] is valid.

As mentioned in the section 2, at high pressures and high pulse repetition rate a jitter at the light pulses was observed while no simultaneous jitter at the actual starting voltage was observed. This may be attributed to local fluctuations in the electron and ion concentration observed mainly at high pressures [15]. These cause random fluctuations in the current and consequently in the laser optical output.

\section{Conclusion.}

In the domain of the optical peak power three regions are distinguished with respect to the performance of the $\mathrm{N}_{2}$ laser.

In the region I the peak power of the pulses presents stability and reliability while in the region II the peak power of the pulses is nearly double with regard to the ones in the region I, with a jitter of about $15 \%$. Finally in the region III break down in the tube does not occur and the $\mathrm{N}_{2}$ laser stops to emit light pulses.

This behaviour of the $\mathrm{N}_{2}$ laser is attributed to different ion decay process predominated in each region.

Concretely in the region I the predominant ion decay process is the diffusion while in the regions II and III the predominant one is the recombination.

These conclusions are extracted from our experimental results and theoretical calculations.

Also the ambipolar diffusion coefficient can be estimated.

\section{Acknowledgments.}

The author wishes to thank warmly Prof. R. Rigopoulos for his interest and stimulating discussions, Dr. B. Giannetas for his assistance with the measurements taken and S. Koutsouvelis for his ample technical assistance during this research.
[1] WoodWARd, B. W. et al., Rev. Sci. Instrum. 44 (1973) 882-887.

[2] Giannetas, B. et al., Revue Phys. Appl. 20 (1985).

[3] Persefhonis, P. et al., Revue Phys. Appl. 18 (1983) 295-300.

[4] Persephonis, P. et al., Revue Phys. Appl. 18 (1983) 731-735.

[5] Mrtani, T. et al., J. Phys. D : Appl. Phys. 11 (1978) 2071-2081.

[6] Mitani, T., J. Appl. Phys. 52 (1981) 3159-3166.

[7] Fitzsimmons, W. A. et al., IEEE J. Quantum Electron QE-12 (1976) 624-633.

[8] BIONDI, M. A., Phys. Rev. 129 (1963) 1181-1188.

[9] Hackam, R. et al., Proc. Phys. Soc. 84 (1964) 133139.
[10] Wilhelm, H. E., J. Chem. Phys. 53 (1970) 16771682.

[11] BIOndi, M. A., Phys. Rev. 83 (1951) 1078-1080.

[12] Kretschmer, C. B. et al., J. Appl. Phys. 34 (1963) 3209-3217.

[13] KaSNer, W. H. et al., Phys. Rev. 137 (1965) A 317-A 329.

[14] Kasner, W. H., Phys. Rev. 164 (1967) 194-200.

[15] Howatson, A. M., An Introduction to gas discharges, 2nd Edition (Pergamon Press) 1976.

[16] NASSER, E., Fundamentals of gaseous ionization and Plasma electronics (John Wiley and Sons, Inc.) 1971.

[17] Cherrington, B. E., Gaseous electronics and gas lasers (Pergamon Press) 1979.

[18] Faire, A. C., et al., Phys. Rev. 113 (1959) 1-6. 